\title{
PROPERTIES OF THE SURFACE BRIGHTNESS DISTRIBUTION OF THE COSMIC X-RAY BACKGROUND
}

\author{
G. De Zotti ${ }^{1}$, A. Franceschini ${ }^{1}$, M. Persic ${ }^{2}$, L. Danese ${ }^{1}$, \\ E. A. Boldt', F. E. Marshall ${ }^{2}$, and G. G. C. Palumbo ${ }^{3}$ \\ ${ }^{1}$ Osservatorio Astronomico and Dipartimento di Astronomia, Padova, Italy \\ ${ }^{2}$ Laboratory for High Energy Astrophysics, NASA/GSFC, Greenbelt, Maryland, USA \\ ${ }^{3}$ Dipartimento di Astronomia, Bologna, Italy
}

\section{THE DATA}

The HEAO-1 A2 scanning survey has provided small field-of-view data characterized by a full width zero intensity of $3^{\circ}$ along the scan direction (at approximately constant ecliptic longitude $\lambda$ ) and of $6^{\circ}$ perpendicular to it. We have used independent fields taken from scanning circles separated by $\Delta \lambda=6^{\circ}$ and whose centers are separated by $\Delta \beta=3^{\circ}$. After having excluded all fields at $|\beta|<60^{\circ}$ or at $|b| \leq 20^{\circ}$, only 905 of the total turned out to be not significantly contaminated by diffuse galactic emission or by known discrete sources. The flux intensity at each field's central position was evaluated by fitting a point source plus a constant background. The sample average $C(\theta)$ of the products $d I(\theta) \times d I^{\prime}(\theta+\Delta \theta)$ of positive and negative intensity fluctuations computed for angular separations of $3^{\circ}, 6^{\circ}, \ldots$, and $27^{\circ}$ provides the $\mathrm{X}$-ray background (XRB) angular correlation function.

No significant signal has been detected on any angular scale. A conservative estimate of the upper limits to $\Gamma(\theta)=[C(\theta)]^{1 / 2} /<I>$ has been found by simply taking the ratio of the observed standard deviation to the square root of the number of field pairs: the $95 \%$ confidence limits amount to $\cong 2 \times 10^{-2}$ for $\theta=3^{\circ}$ and slightly higher values for larger angular scales (Persic et al. 1989). Tighter constraints are expected from fitting the predicted probability distribution to the data and after allowing for all "local" effects that broaden the observed distribution.

The cell-to-cell intensity fluctuations $\langle\Delta / I I\rangle$ of the XRB have been analyzed by Shafer and Fabian (1983). The observed $\langle\Delta I / I\rangle$ can be entirely accounted for by Poisson fluctuations in the space distribution of known classes of sources. The $90 \%$ confidence upper limit to any additional contribution on a scale of 26 square degrees is $2.3 \%$.

\section{RESULTS}

The above limits to the XRB intensity variations can be used to constrain the large-scale distribution of hard X-ray emitters. The predicted contributions of various classes have been computed under the simplifying assumptions that the luminosities are independent of positions and that the two-point spatial correlation function can be represented by a simple power law of constant shape

$$
\xi(r, z)=\xi_{0}(r)(1+z)^{-(3+\varepsilon)},
$$

S. Bowyer and C. Leinert (eds.), The Galactic and Extragalactic Background Radiation, 410-411.

(c) 1990 IAU. Printed in the Netherlands. 


$$
\xi_{0}(r)= \begin{cases}\left(r / r_{0}\right)^{-1.8} & \text { if } r<r_{\text {max }}, \\ 0 & \text { if } r \geq r_{\max },\end{cases}
$$

as a function of the clustering length $r_{0}$. The cutoff radius $r_{\max }$ takes into account that $\xi_{0}(r)$ will steepen and eventually become negative at large distances. The last factor in equation (1) models the possible cosmological evolution of clustering. If the number of objects at fixed proper separation $r, n(z) \xi(r, z)$ is constant (statistically stable clustering), then $\varepsilon=0$; if $\xi(r, z)$ is constant in physical coordinates, $\varepsilon=-3$. We summarize below the most relevant results of our analysis (see also De Zotti et al. 1989).

Galaxy clusters and superclusters. The HEAO-1 A2 bounds on $\Gamma(\theta)$ are compatible with the Bahcall and Soneira (1983) estimate of the correlation-length scale for rich clusters. Evolution of the kind predicted by Kaiser (1986) is also allowed, provided that the gas temperature decreases significantly with increasing $z$. The predicted $\Gamma(\theta)$ would however increase significantly if superclusters are correlated as strongly as suggested by Bahcall and Burgett (1986). In this case, important constraints on evolution models would ensue and an upper limit of $10 \%$ would be set to the integrated contribution of supercluster X-ray emission to the XRB.

Active galactic nuclei (AGNs). Optical evidence for clustering of quasi-stellar objects (QSOs) with an average redshift $z \cong 1$ has been found, among others, by Iovino and Shaver (1988). The estimated amplitude of the quasar correlation function is $\xi \approx 1$ on a proper scale $\cong 10 \mathrm{Mpc}$. This amplitude would correspond to a present clustering length $r_{o} \approx 30 \mathrm{Mpc}$ for stable clustering $(\varepsilon=0)$ or to $r_{o} \approx 10 \mathrm{Mpc}$ if the spatial correlation function $\xi(r)$ is constant in physical coordinates $(\varepsilon=-3)$.

The latter possibility is compatible with the X-ray upper limits on $\langle\Delta I / I\rangle$ and on $\Gamma\left(3^{\circ}\right)$, independently of the assumed value of the density parameter $\Omega$ and of the evolution model for the luminosity function. On the other hand, some data have been reported (Iovino and Shaver 1988) indicating that the amplitude of the quasar-quasar correlation function is decreasing with increasing $z$, perhaps as fast as implied by the stable clustering model. In that case, the predicted intensity variations for pure-luminosity evolution would exceed the observational limits. These constraints could be somewhat weakened if low-luminosity AGNs would be less clustered than high-luminosity objects.

Cluster-AGN correlations. Cluster-AGN correlations depend strongly on the evolution models for AGNs. In the case of pure luminosity evolution, we find $r_{o} \leq 70-80 \mathrm{Mpc}$ (depending on $\left.r_{\max } / r_{o}\right)$ if $\varepsilon=0$, and $r_{o} \leq 45-55 \mathrm{Mpc}$ if $\varepsilon=-3$. If only high luminosity AGNs $(\log L$ $>43.5$ ) are allowed to evolve, the constraints are much weaker. Note that these limits are the only ones available so far.

\section{REFERENCES}

Bahcall, N. A. and Burgett, W. 1986, Ap. J. (Letters), 300, L15.

Bahcall, N. A. and Soneira, R. M. 1983, Ap. J., 270, 20.

De Zotti, G. et al. 1989, Ap. J., submitted.

Iovino, A. and Shaver, P. A. 1988, Ap. J. (Letters), 330, L13.

Kaiser, N. 1986, MNRAS, 222, 323.

Persic, M. et al. 1989, Ap. J. (Letters), 336, 147.

Shafer, R. A. and Fabian, A. C. 1983, in IAU Symposium 104, Early Evolution of the Universe and Its Present Structure, ed. G. O. Abell and G. Chincarini (Dordrecht: Reidel), p. 333. 